\title{
"BUS LANE WITHIN THE AREA OF INTERSECTION" METHOD FOR BUSES PRIORITY ON THE INTERSECTIONS
}

\author{
Ihor Vikovych ${ }^{1}$, Roman Zubachyk ${ }^{2}$ \\ Department of Transport technology \\ Lviv National Polytechnic University \\ Lviv, Stepana Bandery street, 12, Ukraine, 79013 \\ E-mails: ${ }^{1}$ wikovigor@gmail.com; ${ }^{2}$ roman.zubachyk@gmail.com
}

\begin{abstract}
The primary objective of this article is to formalize the "special bus lanes within the area of intersection" method that allows providing buses with space-time priority at signalized intersections (mostly of the isolated type), including those with no more than two traffic lanes in each direction at the approaches to the intersection. The article establishes the limits for efficient application of this method, and describes the results of a simulation experiment conducted in the VISSIM environment to investigate the functioning of the method on an actual intersection. The most critical phase of implementation of this method is to determine the optimum length of the special bus lane at the approach to the intersection. The optimum length of special bus lanes at the approaches to isolated or coordinated intersections is determined based on the maximum length of queued vehicles which is computed using the simulation models developed in the Objective-C language. The article covers the basic characteristics of those models, their structure and building principles, and also provides the model validation results. Simulation models can be used both for determination of the optimum length of special bus lanes at the approaches to signalized intersections and for analysis of intersection performance based on the maximum length of queued vehicles.
\end{abstract}

Keywords: bus lanes, bus signal priority, priority at the intersection, maximum length of queued vehicles

\section{Introduction}

Today the search for new ways to reduce the street network load in the cities is as important as ever. This problem can be addressed either by redesigning such networks or by streamlining their operations through use of Intelligent Transportation Systems (ITS). However, these two approaches aren't always effective in urban conditions. The first one is time-consuming, requires significant capital investments and depends on the functional characteristics of the street network, and the second one doesn't always produce the desired results because it simply optimizes the situation instead of resolving it on a fundamental level. There is, however, an alternative in the form of a third approach which focuses on efficient operation of public transport. Ultimately, it allows reducing the usage of personal vehicles which account for a dominant share in the existing traffic flows and, therefore, decreasing the street network load.

Out of all types of public transport currently used in Ukrainian cities, the urban commuter buses (trolleybuses) are in critical need of development, since they are the most common mode of passenger transport used by the urban population. Giving them priority in traffic at signalized intersections is one of the primary factors that can improve the transport service within the urban street network, since such intersections cause the most significant delays.

Bus rapid transit, (2007) mentions that, in order to ensure priority for buses at signalized intersections, the special bus lanes allocated within the street segments should not be interrupted at the intersections, allowing buses to cross them in the same phase as non-priority traffic flows. Passage of buses in the same phase with non-priority traffic flows can be implemented by prohibiting certain types of turns at the intersection (usually left or right turns) or arranging bypasses (such as a channelizing island bypass, loop-shaped left turn in the intersection area or U-turn outside the intersection area). The intersection conditions do not always allow using such methods without altering the signal control at the intersection. Therefore, they are generally utilized at those intersections where buses cross the intersection in the forward direction, and the non-priority traffic flows making the turns are of low intensity and, therefore, can be prohibited.

Skabardonis (2000), Przhibyl, Svitek (2003), Lin at al. (2015) propose methods of providing passive and active priority for buses. Passive priority is implemented by increasing the green time duration (Shelkov, 1995), reducing the cycle length (Bus rapid transit, 2007) or introducing an additional phase (Klinkovshtein, Afanasiev 2001; Kremenets, Pechersii 2005). It is suggested to increase the green time duration in the direction of bus travel in cases where there is no bus traffic in other directions. Such approach improves the traffic capacity in the target direction and raises the probability of buses crossing the 
intersection during the green time. Reduced cycle length is used when the conditions at the intersection allow prohibiting certain maneuvers for non-priority traffic flows. This allows using fewer phases and, therefore, making the inefficient part of the cycle (number and total duration of intergreen times) shorter, thus reducing the amount of delays both for non-priorty traffic flows and for buses. Introduction of an additional bus phase depends on the intensity of bus traffic in the given direction. A disadvantage of such additional phases is that they increase the total duration of intergreen times, making them generally undesirable in terms of optimizing the traffic control at the intersection.

All active priority methods are implemented by detecting buses at the approach to the intersection using special detectors and altering the signal control modes accordingly. To implement active priority, Angus (2001), Ma, Yang (2008), Ekeila at al. (2009) propose a green time extension algorithm triggered when a bus approaches the intersection at the end of the green time. To provide priority for the buses arriving at the intersection during the red time, Shelkov (1995), Garrow, Machemehl (1997), Wie at al. (2013) suggests using an early red time termination algorithm. This algorithm is especially difficult to implement when there is intensive pedestrian traffic during the phase that needs to be terminated early. Balke at al. (2000), Przhibyl, Svitek (2003), Lin at al. (2015) believe that the most efficient way of providing active priority is to invoke a special phase that can be activated at any time during the cycle length. When using such an algorithm, efficient signal control at the intersection is maintained through so-called "compensation" techniques: after a bus is granted priority passage with the help of the special phase, the time lost by non-priority traffic flows is compensated by increasing the green time duration in their respective directions.

Vikovych, Zubachyk (2013a) refer to all passive and active priority algorithms as time-priority methods, and to those described above - as space-priority methods. They also define a separate group of space-time priority methods. Use of special bus lanes and corresponding adaptation of the traffic light signal control algorithms are considered to be integral components of space-time priority.

To provide space-time priority, Scnabel (1997) proposes introducing bus "gates". This method is based on placing two traffic lights at the approach to the intersection, with the space between them forming a "gate". When the red signal is active at the second traffic light, the "gate" opens for buses traveling in the special bus lane. As an additional benefit, the buses can easily complete turning maneuvers regardless of the location of the special bus lane on the traffic way (leftmost or rightmost lane). When the green time begins, the buses become the first vehicles to cross the intersection in the target direction. This method should be implemented in cases when there are no stops in the intersection area, and is efficient only when two-phase signal control is used.

The (Abdelghany at al., 2007; Example collection, 2010; Ding at al., 2014) proposes using special bus lanes and active priority algorithms to provide space-time priority. Application of these methods produces the best results in terms of ensuring absolute priority (non-stop travel) for the buses.

Individual space and time priority methods can be utilized efficiently only in case of low-intensity bus and non-priority traffic flows. On the other hand, the space-time priority methods can ensure priority passage even at higher traffic (especially bus traffic) loads. The determinant criterion for implementation of methods that provide space-time priority for buses at signalized intersections is the availability of at least three lanes running in the same direction in the streets forming the intersection. Conditions where such priority is difficult to implement include signalized intersections with no more than two traffic lanes in each direction at the approach, which is especially relevant for urban street networks characterized by compact planning. For example, in Lviv (Ukraine) $95 \%$ of the streets have no more than two lanes in each direction.

\section{Background of the space-time prioritization method}

To provide the buses with space-time priority at signalized intersections with two or less approach lanes, the authors propose a method called "special bus lanes within the area of intersection" (Vikovych and Zubachyk, 2013a). This method is based on creating additional expansions in certain directions at the approach to and after the intersection, and arranging a special bus lane for priority traffic within such expansions, including the area of intersection between them. Establishment of such a spatial "corridor" within the intersection area provides free access to the stop line as well as a dominant position in space on the traffic way without reducing the number of approach lanes for non-priority traffic. If there are no stops at the intersection or if stops are located before the intersection, the additional expansions are created at the approaches only.

For isolated X-intersections, six basic types of special bus lanes divided into two groups are proposed to be implemented within the intersection area. The first group is designed for intersections with no stops or stops located before the intersection (Fig. 1a-b - pedestrian crossings not shown), the second 
one - for those cases where stops are located after the intersection (Fig. 1c-f). However, if there are no pedestrian crossings at the intersection, it may sometimes be advisable to implement special bus lanes from the second group at intersections with no stops or stops immediately before them. This would simplify the space-time prioritization and help optimize the operation of the intersection.
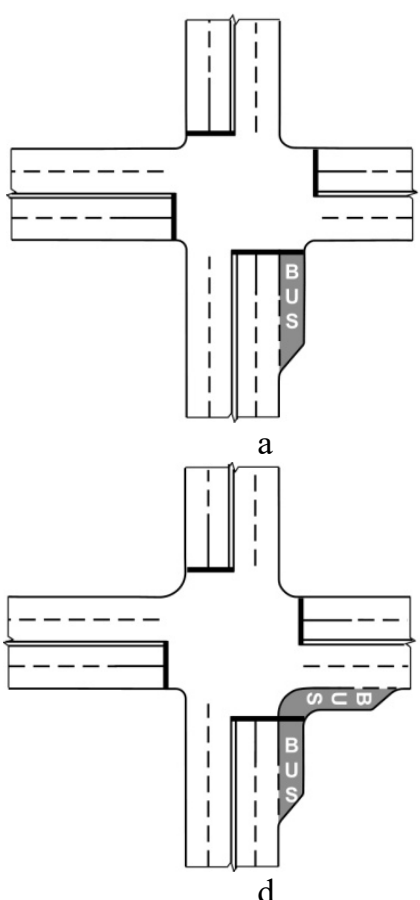

d
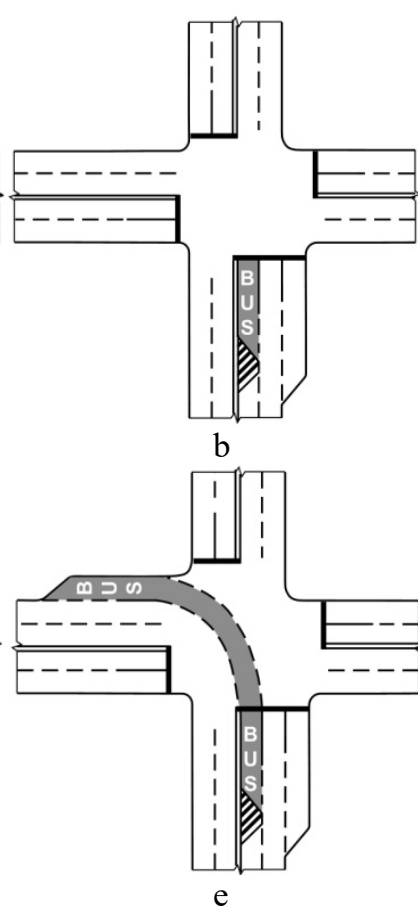

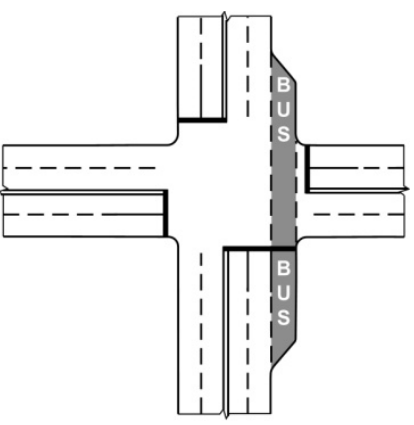

c

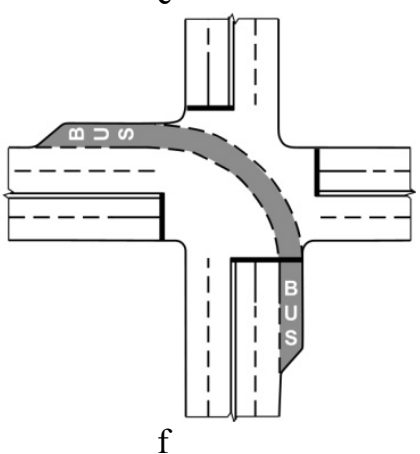

Figure 1. Special bus lane types within the intersection area broken down into two groups: $\mathrm{a}$ - type $1.1 ; \mathrm{b}-1.2 ; \mathrm{c}$ - type $2.1 ; \mathrm{d}-2.2 ; \mathrm{e}-$ type $2.3 ; \mathrm{f}-2.4$

In addition to traffic prioritization at isolated intersections, special bus lanes may also be implemented at intersections operating under a coordinated control system. This is especially relevant for cases where stops are located after two adjacent intersections with coordinated control, and the coordination area covering those intersections has two traffic lanes in one or both directions. In such conditions, delays may occur at the adjacent intersection due to lack of synchronization between the movement of buses from stop to stop and the green time in the coordinated direction. In other words, it is virtually impossible to ensure non-stop bus traffic between the two adjacent stops without disrupting the coordinated control. Therefore, to reduce bus delays at both upstream and downstream intersections, it would be practical to implement special bus lanes of type 2.1 in such areas (Fig. 1c).

One of the key stages of implementing this method is determination of the optimum length of the special bus lane at the approach to the intersection which is calculated based on the maximum length of queued vehicles in the lane adjacent to the special bus lane during the cycle.

\section{Determination of the maximum length of queued vehicles at a signalized intersection}

\subsection{On isolated intersection}

To determine the maximum length of queued vehicles at an isolated intersection which is needed to calculate the optimum special bus lane length at the approach to the intersection, a simulation model of intersection approaches described in (Vikovych and Zubachyk, 2013b) was developed. The model was written in the Objective-C language using the Xcode software environment. It is based on simulation of vehicles arriving at the intersection, waiting in the queue, and departing from the queue during the green time. The main component of this model is the vehicle arrival times expressed as time intervals between the vehicles and distributed according to the Hyper-Erlang and log-normal distribution laws (Inose, Khamada, 1983; Polischuk, Dzyuba, 2008). The Hyper-Erlang distribution density is defined by the following expression: 
$f(x)=\beta \cdot \lambda_{1} e^{-\lambda_{1}(x-\tau)}+(1-\beta) \cdot \frac{\lambda_{2}^{a}}{(a-1) !} x^{a-1} \cdot e^{-\lambda_{2} x}, x \in(0 ;+\infty)$

where $a, \lambda_{1}, \lambda_{2}$ distribution law parameters; $a>0 ; \lambda_{1}>0, \lambda_{2}>0 ; \lambda_{1}=\frac{1}{\bar{x}}, \lambda_{2}=\frac{a}{\bar{x}} ; \bar{x}$ - average time interval between the vehicles; $\tau$ - minimum interval between the vehicles (ranging from 0,5 to $1,5 \mathrm{~s}$ ); $\beta$ - share of vehicles arriving freely at the approach to the intersection.

The log-normal distribution density is defined by the following formula:

$f(x)=\frac{1}{x \cdot \sigma \sqrt{2 \pi}} \cdot e^{-\frac{(\ln x-\mu)^{2}}{2 \sigma^{2}}} \quad x \in(0 ;+\infty)$

where $\mu, \sigma^{2}-$ mean and variance; $\sigma>0, \mu=\bar{x}$.

The simulation model the interface of which is shown in Fig. 2 can also run on mobile iOS devices (iPhone, iPad), and is implemented in such a way so that the user can to easily change the simulation conditions by modifying the input parameters: duration of the simulation period; number of approaches to the intersection $(\leq 4)$ and number of traffic lanes within each approach $(\leq 3)$, including the traffic pattern; geometric approach parameters (traffic lane width, longitudinal gradient, right and left turn radius); traffic control parameters (cycle length, green and yellow time); traffic conditions (traffic intensity, breakdown by primary directions, share of trucks and buses in the flow) etc.

The model produces the maximum length (in vehicles and meters) of the queue formed at the beginning of the green time and during the cycle. The queue length per cycle is the number of vehicles in the queue formed at the beginning of the green time plus the number of vehicles that arrive at the intersection during the dissipation of the queue until the last vehicle departs from the initial queue. The queue length at the beginning of the green time can also be used to evaluate the signal control efficiency, while the queue length per cycle can be used to determine the length of the additional expansion at the approach to the intersection designed to increase the intersection capacity or provide space-time priority for the buses ("special bus lanes within the area of intersection" method).
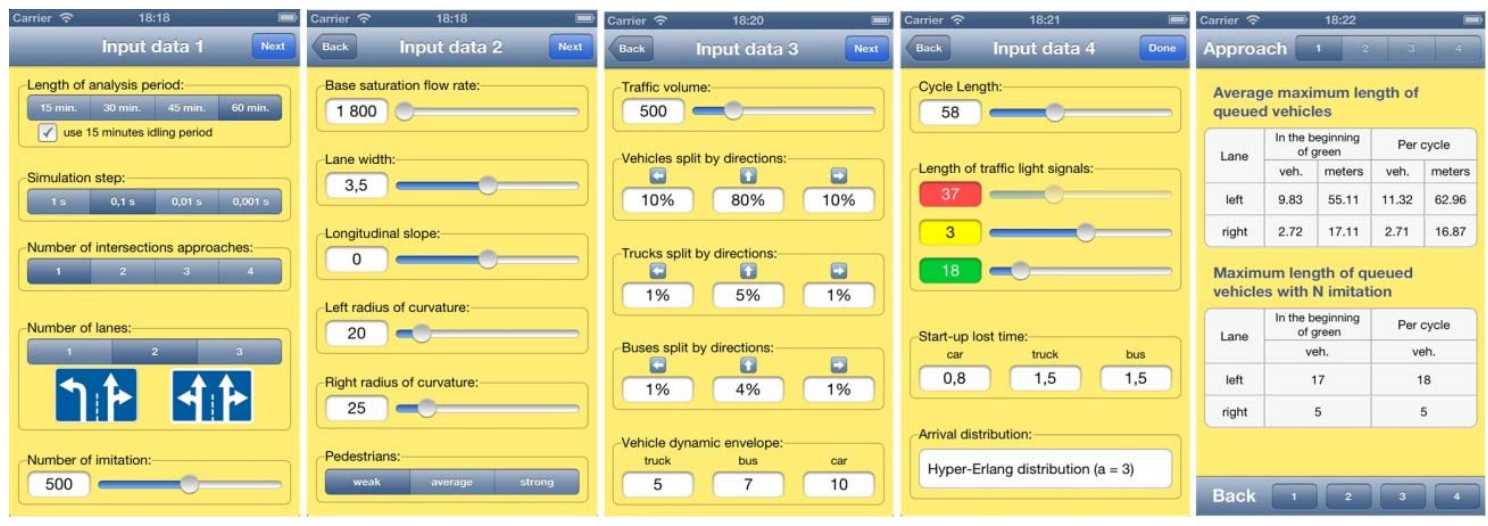

Figure 2. Interface of the model used to determine the length of queued vehicles

Application of this model allowed identifying how the pattern of arrival of vehicles at the intersection affects the maximum length of queued vehicles. If the vehicle arrival times are distributed in the model according to the Hyper-Erlang distribution with $a=3$, the simulated queue values are similar to those obtained using the German standard formula (Manual for the design, 2001) and close to those produced by VISSIM. On the other hand, analysis of the maximum length of queued vehicles showed that the time intervals between the vehicles arriving at the intersection match the actual values if they are distributed according to this law.

Based on the maximum length of queued vehicles generated by this model, the optimum special bus lane length at the approach to an isolated intersection was determined for a homogeneous traffic flow with the car length of $6 \mathrm{~m}$ and flow ratios of $\mathrm{X}_{i}=0,9$ and $\mathrm{X}_{i}=0,95$. The latter corresponds to the upper limit of normal traffic conditions at the intersection approach (Scnabel, 1997). The results are shown in Fig. 3. 


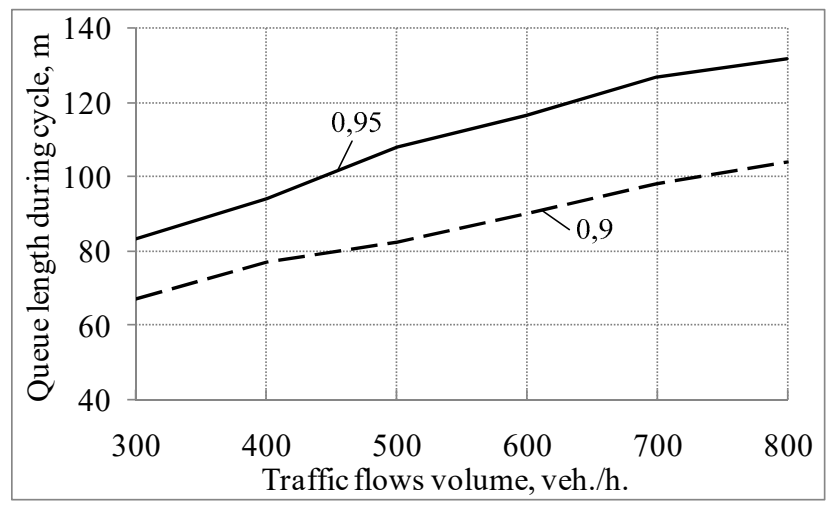

Figure 3. Maximum length of queued vehicles per cycle in meters (numbers on the curves are the flow ratios)

The results provided in Fig. 3 show that the optimum special bus lane length at the approach to an isolated intersection ranges from $59 \mathrm{~m}$ to $135 \mathrm{~m}$.

\subsection{On coordinated intersection}

To determine the maximum length of queued vehicles at an adjacent intersection in the coordinated direction, a simulation model described in (Vikovych and Zubachyk, 2013c) was developed. This model which simulates the operation of a coordinated area for two adjacent intersections was also written in the Objective-C programming language. The input parameters for the model are: duration of the simulation period; upstream intersection type (crossing or junction); coordinated area length; traffic volume at the upstream and secondary approaches with breakdown by traffic lanes, traffic directions and flow composition (cars, trucks, buses); share of vehicles coming from the secondary approaches in the traffic lanes within the coordinated area; cycle, phases and greet time duration at both intersections; coordinated speed.

The output parameters of the model are the average maximum lengths of queued vehicles per $k$ simulations at the adjacent intersection in the coordinated direction for queues formed by vehicles from the upstream approach and secondary approaches as well as their total value.

The model is implemented in such a way so that the user can easily change all input parameters. However, it is suitable only for coordinated street areas with two traffic lanes in one or both directions where the upstream and secondary approaches are isolated and both adjacent intersections have the same cycle length.

The simulation model was created based on the analytical research of the vehicle queue formation process at the adjacent intersection in the coordinated direction described in (Vikovych and Zubachyk, 2013c). According to the research data, the queue at the adjacent intersection is formed by vehicles entering the coordinated area from the upstream approach and secondary approaches.

Whether all or some of the vehicles from the secondary approaches enter the queue depends on the control parameters between the adjacent intersections, including the green time duration in the coordinated direction at both intersections as well as the phase shift value.

In view of the above, the length of the queue formed by vehicles coming from the secondary approaches is determined by the following formula:

$$
Q_{S_{i}}^{k}=\left\{\begin{array}{ll}
\int_{(k-1) \cdot T_{c}}^{k \cdot T_{c}}\left(\lambda_{U}(t) \cdot \beta_{L}+\lambda_{D}(t) \cdot \beta_{R}\right) d t & (k=\overline{1, M}) \\
\int_{(k-1) \cdot T_{c}}^{k \cdot T_{c}}\left(\lambda_{U}^{\prime}(t) \cdot \beta_{L}+\lambda_{D}^{\prime}(t) \cdot \beta_{R}\right) d t & (k=\overline{1, M})
\end{array},\right.
$$

where $Q_{S_{i}}^{k}$ - length of the queue in the $i^{\text {th }}$ lane of the adjacent intersection formed by vehicles coming from secondary approaches $p_{U}$ and $p_{D}$ during the $k^{\text {th }}$ signal control cycle, vehicles; $\lambda_{U}(t)=\frac{N_{U}\left(t_{g}\right)}{T_{c}}$, $\lambda_{U}^{\prime}(t)=\frac{N_{U}^{\prime}\left(t_{g_{\Delta 2}}\right)}{T_{c}}, \lambda_{D}(t)=\frac{N_{D}\left(t_{g}\right)}{T_{c}}$, and $\lambda_{D}^{\prime}(t)=\frac{N_{D}^{\prime}\left(t_{g_{\Delta 2}}\right)}{T_{c}}-$ flow ratio in secondary approaches $p_{U}$ 
and $p_{D}$ at $t_{g}$ and $t_{g_{\Delta 2}}$, respectively; $N_{U}\left(t_{g}\right), N_{U}\left(t_{g_{\Delta 2}}\right), N_{D}\left(t_{g}\right), N_{D}\left(t_{g_{\Delta 2}}\right)$ - exit flow volume in the secondary approaches during $t_{g}$ and $t_{g_{\Delta 2}}$, respectively; $t_{g}$ - green time duration; $t_{g_{\Delta 2}}$ - green time period during which the vehicles join the queue at the adjacent intersection (determined by the formulas provided in (Vikovych and Zubachyk, 2013c); $\beta_{L}$ and $\beta_{R}$ - share of vehicles arriving from secondary approaches $p_{U}$ and $p_{D}$ to the $i^{\text {th }}$ lane of the adjacent intersection; $T_{c}-$ cycle length; $M=T / T_{c}-$ number of cycles during the simulation period $T$.

The following formula is proposed to determine the length of the queue formed by vehicles coming from the upstream approach:

$Q_{U_{i}}=\left\{\begin{array}{ll}\frac{\left(\tau_{d}+\tau_{\max }-t_{p s}-t_{g_{p 1}}\right) \cdot N_{v g}(t)}{\tau_{d}+\left(\tau_{\max }-\tau_{\min }\right)} & \text { in case } Q_{U_{i}}>0 \\ 0 & \text { in case } Q_{U_{i}}<0\end{array}\right.$,

where $Q_{U_{i}}$ - length of the queue in the $i^{\text {th }}$ lane of the adjacent intersection formed by vehicles coming from the upstream approach, vehicles; $\tau_{\min }, \tau_{\max }$ - time of travel through the coordinated area for the first and last vehicle in the group, respectively; $\tau_{d}=N_{v g}(t) \cdot h_{p_{0}}$ - duration of vehicle group dissipation at the upstream approach; $N_{v g}(t)$ - number of vehicles in the group at the upstream approach; $h_{p_{0}}$ - average interval between the vehicles during the queue dissipation at the upstream approach $p_{0} ; t_{p s}-$ phase shift duration; $t_{g_{p 1}}$ - green time duration at the adjacent intersection in the coordinated direction.

Formula (4) is based on the time it takes for the vehicles in the group to travel through the coordinated area which is distributed normally (normal distribution) in the simulation model according to the following expression:

$\tau_{i}=a+\sqrt{2 \sigma^{2}} \cdot \operatorname{erf}^{-1}\left(2 y_{i}-1\right) \quad y_{i} \in(0 ; 1)$

where $a$-time of travel through the coordinated area at the coordinated speed; $\sigma$-standard deviation of the vehicle travel time in the coordinated area.

The standard deviation of the vehicle travel time in the coordinated area depends on whether each vehicle in the group maintains the recommended speed while passing through the coordinated area, which, in turn, is influenced by many factors. The value of $\sigma$ is primarily affected by the length of the coordinated area, number of vehicles in the group, group composition, and share of turning maneuvers from the upstream intersection. The influence of these factors on the vehicle travel time in the coordinated area was determined using the VISSIM software. As a result of the simulation, the corresponding values of the $\sigma$ were obtained, and the nature of their dependence on the above factors was identified.

In the simulation model, the standard deviation of the vehicle travel time in the coordinated area is determined using the following equation:

$\sigma=0,9281-0,0256 N_{v g}+0,0009 N_{v g}^{2}+0,6214 L+2,5695 L^{2}+0,0332 N_{t}-0,2363 \beta_{S T}$

where $N_{v g}$ - number of vehicles in the group in a single lane; $L$ - coordinated area length, km; $N_{t}-$ number of trucks in the group; $\beta_{S T}-$ straight flow share.

Application of this model showed that it capable of accurately reproducing the actual processes occurring at the adjacent intersection, and allows determining the maximum length of queued vehicles with the results closely matching the values generated by VISSIM (deviation does not exceed 22\%). The largest deviations were observed in cases with a relatively small share of homogeneous flow and long coordinated $\operatorname{area}(500 \mathrm{~m}$ and more). The adequacy of these results proves the hypothesis that the length of the queue formed by the vehicles from the upstream approach is directly proportional to the size (measured in time) of the group that arrives at the adjacent intersection during the red time, and inversely proportional to the average interval between the vehicles in the group. 
The most important results of this research are not the calculated values of the actual maximum length of queued vehicles, since those can be easily determined using the currently available software, but rather the analytical description of the queue formation process at the adjacent intersection and the newly developed algorithm for computation of the maximum queue length through simulation modeling.

\section{Analysis of performance of the "Special bus lanes within the area of intersection" method in terms of time prioritization}

To ensure proper functioning of the "special bus lanes within the area of intersection" method, it is crucial to supplement it with effective means of providing priority passage through the intersection area which is achieved with the help of traffic light signal control algorithms, first of all - of the adaptive type. Algorithms that rely on green time extension or early red time termination upon arrival of a bus at the approach are suitable only for special bus lanes belonging to the second group, and only in cases where buses and non-priority traffic flows can cross the intersection within the same phase without any conflicts. The special phase activation algorithm allows providing absolute and conditional priority for all types of special bus lanes in the intersection area. However, it can create problems for other road users, and its efficiency depends on the traffic conditions at each approach as well as the interval between the buses arriving at the intersection.

Efficiency of this algorithm in combination with a special bus lane in the intersection area under different traffic conditions was verified in the VISSIM environment using a model of an X-shaped signalized intersection with a single traffic lane at all approaches in both directions and with a type 2.1 special bus lane 2.1 (Vikovych at al., 2014). The simulation showed that changes in the approach conditions expressed quantitatively through the flow ratio affect the average vehicle delay at the approach. In particular, the delays become longer as the approach flow ratio increases and the interval between the buses decreases (Fig. 4).

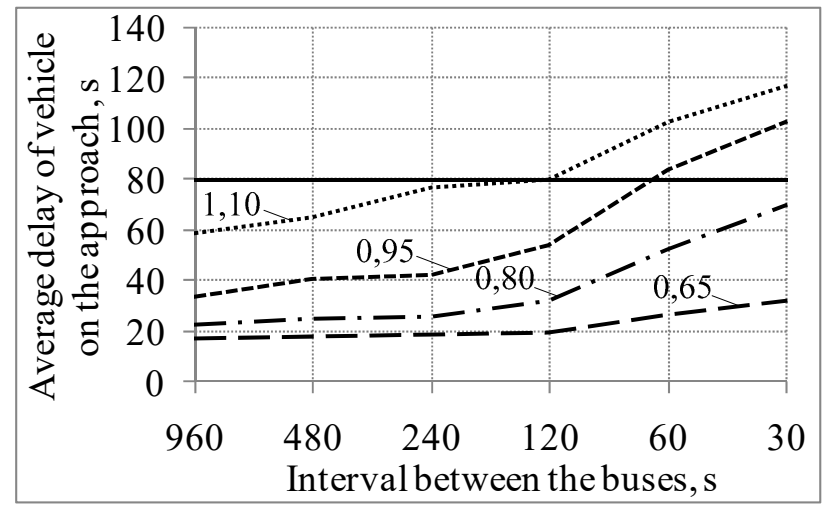

Figure 4. Dependence of the average approach delay for other vehicles on the interval between the buses (numbers on the curves are the approach flow ratios)

The effective range for application of the "special bus lanes within the area of intersection" method was determined based on the above findings using regression analysis subject to the upper limit of the average vehicle approach delay equal to $80 \mathrm{~s}$ in accordance with the American regulations (Highway Capacity Manual, 2000). The results show that, for example, the interval between the buses should be at least $57 \mathrm{~s}$ provided that the normal traffic conditions at each approach are not exceeded (flow ratio of 0,95 or below).

These results enable the engineers to easily determine the feasibility of implementation of the "special bus lanes within the area of intersection" method at intersections where time priority is provided using the adaptive special phase activation algorithm.

\section{Testing of the research results}

To verify the "special bus lanes within the area of intersection" method under actual conditions, the isolated signalized X-intersection between Mazepy and Mykolaichuka Streets (Lviv) with one traffic lane in each direction at each approach was used as an example. 13 bus routes and 1 trolleybus route pass through 
this intersection in different directions. The longest interval between the buses traveling through the intersection is $24 \mathrm{~m}$, while the shortest interval is $65 \mathrm{~s}$.

To reduce the bus delays at this intersection, it is proposed to implement the following special bus lanes in the intersection area: type 2.1 on Mazepy Street in both directions, and types 2.2 and 2.4 at one of the approaches on Mykolaichuka Street (Fig. 5a).

The length of the special bus lane elements in the intersection area, including the optimum length of the special bus lane element at the approach, is to be determined using the method described above.

Time priority is to be provided using an algorithm based on special phase activation. At the same time, to ensure optimum control of non-priority traffic flows at the intersection, it is proposed to combine this algorithm with a time gap search algorithm and a variable phase alternation algorithm. The ways of combining signal groups into phases and possible phase alternation options are shown in Figure 5b.

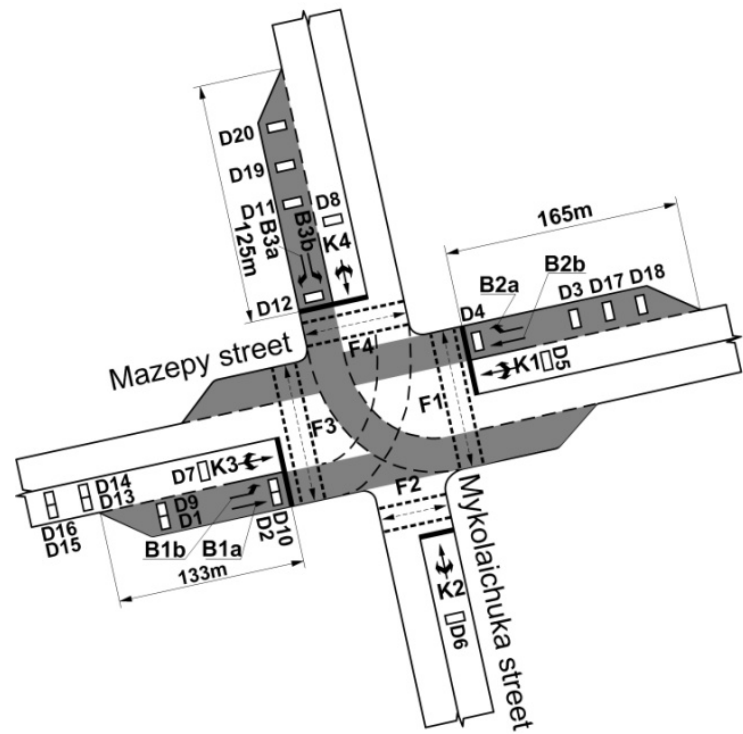

a

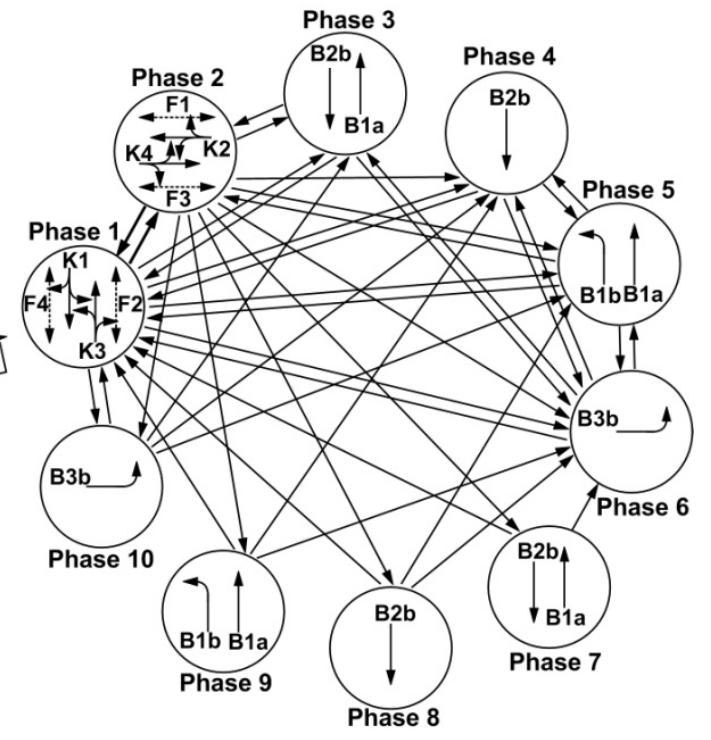

$\mathrm{b}$

Figure 5. Proposed changes to the intersection between Mazepy and Mykolaichuka Streets: a - geometric parameters of the intersection with special bus lanes added to the intersection area; $b$ - phase alternation sequence

The efficiency of these proposals is verified by creating a simulation model of the intersection between Mazepy and Mykolaichuka Streets in the VISSIM software environment. The vehicle and pedestrian traffic volume values corresponding to the existing conditions at the intersection during the rush hours are entered into the model. It is assumed that the desired speed of cars and trucks is $50 \mathrm{~km} / \mathrm{h}$, while the desired speed of buses and trolleybuses is $40 \mathrm{~km} / \mathrm{h}$.

Eight phases (3 through 10) are used to provide the buses with priority passage through the intersection by activating a special phase. These phases are skipped if there are no buses at the approaches (Fig. 5b). Phases 4, 6, 7, 8, 9 and 10 are always followed by phase 1 (unless they were activated), while phases 3 and 5 may change either to phase 1 or to phase 2 .

Arrival of buses from signal group B1a is detected by means of detectors D1, D2, D13 and D15; signal group B1b - D9, D10, D14 and D16; signal group B2b -D3, D4, D17 and D18; signal group B3b D11, D12, D19 and D20 (Fig. 5a). Buses from signal groups B2a and B3a are not detected upon arrival, since they do not require time priority to be given by traffic light signals (as they turn right from the special bus lane). Detectors D5, D6, D7 and D8 are used to identify time gaps in non-priority traffic flows belonging to signal groups $\mathrm{K} 1, \mathrm{~K} 2, \mathrm{~K} 3$ and $\mathrm{K} 4$, respectively.

The model uses the average approach delay for buses and other vehicles and queue length (average and maximum) at the intersection as the criteria for quantitative evaluation of efficiency of the above proposals.

In addition, to enable comparison of these results with those that can be obtained with no priority passage through the intersection, an additional model of the same intersection with the same input data, but without the special bus lanes in the intersection area was created in the VISSIM environment. In this case, the traffic light signals operate in the adaptive mode using the traffic flow gap search algorithm for all approaches with dual-phase vehicle and pedestrian traffic flow dissipation (similar to phase 1 and 2 in 
Figure 5b). The average delay and queue length simulation results for both scenarios are provided in Tables 1 and 2.

Table 1. Intersection performance indicators with implemented space-time prioritization

\begin{tabular}{|l|l|l|l|}
\hline \multirow{2}{*}{ Traffic flows of signal groups } & \multirow{2}{*}{ Average delay, $\mathbf{s}$} & Queue length, veh. \\
\cline { 3 - 4 } & & average & maximal \\
\hline K1 & 70,02 & 9,3 & 22,5 \\
\hline K2 & 69,83 & 7,8 & 22,1 \\
\hline K3 & 29,03 & 3,2 & 14,2 \\
\hline K4 & 79,71 & 7,1 & 23,0 \\
\hline B1 & 3,91 & - & - \\
\hline B2 & 4,85 & - & - \\
\hline B3 & 6,07 & - & - \\
\hline
\end{tabular}

Table 2. Intersection performance indicators with adaptive control (no prioritization)

\begin{tabular}{|l|l|l|l|l|}
\hline \multirow{2}{*}{ Traffic flows of signal groups } & \multicolumn{2}{|l|}{ Average delay, } & \multicolumn{2}{l|}{ Queue length, veh. } \\
\cline { 2 - 5 } & vehicles (without buses) & buses & average & maximal \\
\hline K1 & 63,28 & 66,24 & 8,6 & 20,4 \\
\hline K2 & 53,16 & 71,30 & 5,4 & 17,8 \\
\hline K3 & 27,69 & 21,77 & 2,7 & 13,7 \\
\hline K4 & 60,39 & 58,28 & 5,7 & 19,5 \\
\hline
\end{tabular}

Tables 1 and 2 show that implementation of space-time priority at the selected intersection allows reducing the bus delays by 82,93 and $90 \%$ for routes belonging to signal groups B1, B2 and B3, respectively. At the same time, this prioritization results in a minor increase in the delay values and queue length for other vehicles. In particular, vehicle delays in signal groups K1, K2, K3 and K4 are increased by $11,31,5$ and $32 \%$, respectively; average queue length is increased by $8,44,19$ and $25 \%$, respectively; and maximum queue length is increased by $10,24,4$ and $18 \%$, respectively.

The average bus delay at the intersection with implemented space-time prioritization is reduced by $87 \%$ compared to adaptive control with no prioritization (from 47,22 to 6,24 s), and the average delay for other vehicles is increased by $19 \%$ (from 50,53 to $60,07 \mathrm{~s}$ ).

Significant reduction of the bus (trolleybus) delays at the intersection allows reducing their route circulation time and fuel consumption, which ultimately improves the efficiency and comfort of passenger transportation.

\section{Conclusions}

This article formalizes the "special bus lanes within the area of intersection" method used to provide buses with space-time priority at signalized intersections (mostly of the isolated type). The results of a simulation experiment conducted in the VISSIM environment show that application of this method at an actual isolated signalized intersection allows achieving an $87 \%$ decrease in the average bus delay compared to non-prioritized adaptive control. This proves that the "special bus lanes within the area of intersection" method can be efficiently used in practice, especially at signalized intersections with no more than two traffic lanes in each direction at the approaches.

The most critical phase of implementation of this method is to determine the optimum length of the special bus lane at the approach to the intersection based on the maximum length of queued vehicles. The article covers the characteristics of the simulation models designed to calculate the maximum length of queued vehicles at the approach to isolated and coordinated intersections.

The simulation model developed for determination of the maximum length of queued vehicles at the approach to an isolated intersection differs from the existing models in that it uses hyper-Erlang law for distribution of time intervals between the vehicles arriving at the intersection. This improves the adequacy of the simulation results (queue values are similar to those obtaining using the German standard formula (Manual for the design, 2001) and close to those produced by VISSIM). The way the model is implemented allows using it on portable devices (iPhone, iPad) and easily changing the simulation conditions by modifying the input parameters.

The simulation model used for calculation of the maximum length of queued vehicles at the approach to a coordinated intersection was created based on the analytical research of the vehicle queue formation process at the adjacent intersection in the coordinated direction. Such research was presented for the first time. 
Both simulation models can be used for determination of the optimum length of special bus lanes at the approaches to signalized intersections as well as for analysis of intersection performance based on the maximum length of queued vehicles.

\section{References}

1. Abdelghany, K. F., Mahmassani, H. S., Abdelghany, A. F. (2007) A Modeling Framework for Bus Rapid Transit Operations Evaluation and Service Planning. Transportation Planning and Technology, vol. 30, issue 6, pp. 571-591.

2. Angus, P. Davol. (2001) Modeling of traffic signal control and transit signal priority strategies in a microscopic simulation laboratory. Massachusetts institute of technology, 118.

3. Balke, K., Dudek, C., Urbanik II T. (2000) Development and evaluation of intelligent bus priority concept. Transportation Research Record: Journal of the Transportation Research Board, vol. 1727, pp. 12-19.

4. Bus rapid transit / Planning guide (2007) New York.: 3ed edition, 825.

5. Ding, L., Zhang, N., Qian, Z. D. (2014) Analysis of Managed Bus Lane Strategies Based on Microcosmic Traffic Simulation. Advanced Materials Research, vol. 1079-1080, pp. 440-447.

6. Ekeila, W., Sayed, T., Esawey, M. E. (2009) Development of dynamic transit signal priority strategy. Transportation Research Record: Journal of the Transportation Research Board, vol. 2111, pp. 1-9.

7. Example collection about the guidelines for traffic signal systems (2010). Research society for roads and Transportation, Cologne, 92.

8. Garrow, M., Machemehl R. (1997) Development and evaluation of transit signal priority strategies. Center for Transportation Research, The University of Texas at Austin, 147.

9. Highway Capacity Manual (2000) TRB, Washington, DC, 1134 p.

10. Inose, H., Khamada, T. (1983) Road traffic control. Moscow, USSR, Transport, 248.

11. Klinkovshtein, H. I., Afanasiev, M. B. (2001) Traffic management. Moscow, Transport, 247.

12. Kremenets, Y. A., Pechersii, M. B. (2005) Technical means of traffic management. Moscow, 279.

13. Lin Y., Yang X., Zou N., Franz M. (2015) Transit signal priority control at signalized intersections: a comprehensive review. Transportation Letters: the International Journal of Transportation Research, vol. 7, issue 3, pp. 168-180.

14. Manual for the design of road traffic facilities (2001) Federal Highway Research Institute. October, 370.

15. Ma W., Yang X. (2008) Efficiency Analysis of Transit Signal Priority Strategies on Isolated Intersection. Journal of System Simulation, issue 12, pp. 184-191.

16. Przhibyl, P., Svitek, M. (2003). Telematic in transport (translation from Czech). Moscow, 540.

17. Polischuk, V. P., Dzyuba, O. P. (2008) Theory of traffic flow: models and methods of traffic management. Kyiv, 175.

18. Shelkov, Y. D. (1995) Traffic management in the cities. Moscow, 143.

19. Scnabel, W. (1997) Fundamentals of traffic engineering and transport planning. Volume 1: Traffic Systems, 2nd edition, Berlin, publisher of Construction mbH, 590.

20. Skabardonis, A. (2000) Control Strategies for Transit Priority. Transportation Research Record: Journal of the Transportation Research Board, vol. 1727, pp. 20-26

21. Vikovych, I. A., Zubachyk R. M. (2013a) Development of method for bus priority on the intersection. Eastern-European Journal of Enterprise Technologies. Scientific Journal, no. 6/4 (54), pp. 28-34.

22. Vikovych, I. A., Zubachyk R. M. (2013b) Development of simulation model for determination of the maximum length of queued vehicles. Herald of the National Technical University "Kharkiv Polytechnic Institute”, Kharkiv, no. 65, pp. 27-33.

23. Vikovych, I. A., Zubachyk R. M. (2013c) Simulation model development for determination of the maximum length of queued vehicles on adjacent direction to coordinated intersection. "Technology audit and production reserves" journal. Scientific Journal, no. 6/1(14), pp. 19-26.

24. Vikovych, I. A., Zubachyk R. M., Bespalov D. O. (2014) Efficiency determination of method "bus lane within the area of intersection" from the standpoint of priority in time. "Technology audit and production reserves" journal. Scientific Journal, no. 5/1(19), pp. 40-45.

25. Wie, L., Zhang, L., Wang, Z. (2013) Cellular automata moderl on bus signal priority strategies considering resource constraints. Practical application of intelligent systems: Proceedings of the eighth international conference on intelligent systems and knowledge engineering, Shenzhen, China, pp. 689 -706 . 